\title{
System performance analysis of hybrid time-power switching protocol of EH bidirectional relaying network in amplify-and- forward mode
}

\author{
Phu Tran Tin", Minh Tran², Tan N. Nguyen ${ }^{3}$, Thanh-Long Nguyen ${ }^{4}$ \\ ${ }^{1}$ Faculty of Electronics Technology, Industrial University of Ho Chi Minh City, Ho Chi Minh City, Vietnam \\ ${ }^{2}$ Optoelectronics Research Group, Faculty of Electrical and Electronics Engineering, Ton Duc Thang University, Ho Chi \\ Minh City, Vietnam \\ ${ }^{3}$ Wireless Communications Research Group, Faculty of Electrical and Electronics Engineering, Ton Duc Thang \\ University, Ho Chi Minh City, Vietnam \\ ${ }^{4}$ Center for Information Technology, Ho Chi Minh City University of Food Industry, Ho Chi Minh City, Vietnam
}

\begin{tabular}{|c|c|}
\hline Article Info & ABSTRACT \\
\hline Article history: & \multirow{7}{*}{$\begin{array}{l}\text { In this paper, we investigate system performance in term of throughput and } \\
\text { ergodic capacity of the hybrid time-power switching protocol of energy } \\
\text { harvesting bidirectional relaying network. In the first stage, the analytical } \\
\text { expression of the system throughput and ergodic capacity of the model } \\
\text { system is proposed and derived. In this analysis, both delay-limited and } \\
\text { delay-tolerant transmission modes are presented and considered. After that, } \\
\text { the effect of various system parameters on the proposed system is } \\
\text { investigated and demonstrated by Monte-Carlo simulation. Finally, the } \\
\text { results show that the analytical mathematical and simulated results match for } \\
\text { all possible parameter values for both schemes. }\end{array}$} \\
\hline Received Jun 9, 2018 & \\
\hline Revised Oct 10, 2018 & \\
\hline Accepted Dec 25, 2018 & \\
\hline Keywords: & \\
\hline Decode-and-forward (DF) & \\
\hline Energy harvesting $(\mathrm{EH})$ & \\
\hline
\end{tabular}

Copyright $\odot 2019$ Institute of Advanced Engineering and Science. Ergodic capacity

Power splitting protocol

Relay network All rights reserved.

\section{Corresponding Author:}

Tan N. Nguyen,

Wireless Communications Research Group,

Faculty of Electrical and Electronics Engineering,

Ton Duc Thang University,

Ho Chi Minh City, Vietnam.

Email: nguyennhattan@tdtu.edu.vn

\section{INTRODUCTION}

Energy harvesting $(\mathrm{EH})$ wireless communication has been attracting intense research interests from both academia and industry, as it can substantially prolong the network lifetime, especially for wireless sensor networks with low power nodes. Energy can be harvested from environment resources, but EH from conventional sources, such as solar and wind, may be sporadic or intermittent, which makes it difficult to satisfy the data transmission requirements. One particular energy source is the radio frequency (RF) signal, which can recharge nodes more controllable. Wireless energy transfer (WET) in the network with a helping relay is the most popular technique [1]-[2]. The helping relay with energy harvesting has been proposing and studied in many last papers. In more details, [3] presented energy harvesting in the amplify-and-forward (AF) relaying. In [4], the total energy harvested from multiple sources was optimally allocated among different destinations. In [5], the effect of large-scale network interference on energy harvesting decode-and-forward (DF) was considered. Moreover, the effect of the random location of the relay on DF relaying is investigated in [6] and [7] maximized the achievable throughput of an AF energy harvesting system. Furthermore, a similar problem was studied in [8] for $\mathrm{DF}$ and [9] proposed the maximum achievable throughput of an $\mathrm{AF}$ 
energy harvesting system. From this point of view, energy harvesting relaying network is the hot direction in the communication network and is necessary to develop more and more.

In this work, the hybrid time-power switching protocols for the energy harvesting bidirectional relaying network is presented. In this relaying network, the outage probability and the ergodic capacity are proposed and demonstrated for both delay-limited (DL) and delay-tolerant (DT) transmission modes. The main contributions of the paper are summarized as follows:

a. The system model of the hybrid time-power switching protocols for the energy harvesting bidirectional relaying network is proposed for both delay-limited (DL) and delay-tolerant (DT) transmission modes.

b. The closed-form of the outage probability and ergodic capacity for the system is derived.

c. The influence of the main parameters on the system performance is demonstrated entirely.

The structure of this paper is proposed as follows. The system model of the hybrid time-power switching protocols for the energy harvesting bidirectional relaying network is proposed and presented in section II. Sections III proposed outage probability and ergodic capacity for both delay-limited (DL) and delay-tolerant (DT) transmission modes are investigated and derived, respectively. Section IV provides the numerical results and some discussions. Finally, some conclusions are proposed in section V.

\section{SYSTEM MODEL}

In this section, the system model is presented in Figure 1. In this model, the user is intended to send information to the access point (AP) with the assistance of a relay R and the AP transfer the energy to the user by the helping relay. Moreover, the direct connection between the AP and the user is so weak, hence, the only available communication path, as well as power transfer path, is via the relay $\mathrm{R}$. The relay $\mathrm{R}$ plays both roles of energy relaying from the AP to the user and information forwarding from the user to the AP [10], [11]. All nodes are assumed to operate in half-duplex mode, and decode-and-forward (DF) can be used at the relay for information transfer. Regarding to the channel model, the perfect channel state information (CSI) is available at the relay and the AP. All channels here experience Rayleigh fading and keep constant during each transmission block so that they can be considered as slow fading. The Figure 2 displayed the time-power switching based protocol model. In the first interval time $\alpha T$, the AP transfer energy by RF to the relay $\mathrm{R}, 0 \leq \alpha \leq 1$. After that, the energy transfer from the AP to the $\mathrm{R}$ and the information transmission from the $\mathrm{U}$ to the $\mathrm{R}$ are performed instantaneously in the second interval $\beta T, 0 \leq \beta$ $\leq 1$. Finally, the information transmission process from R to AP and the energy transfer process from the R to $\mathrm{U}$ are made in the remaining interval time $(1-\alpha-\beta) T$. In this model, we assume that the circuit power consumption is negligible as compared to the radiation power, which is reasonable for low-power devices such as sensor nodes. In this system model, $0 \leq \alpha \leq 1$ and $0 \leq \beta \leq 1$. If $\alpha=0$, this scheme becomes a power splitting protocol. If $\beta=(1-\alpha) / 2$ and $\rho=0$ then it becomes the time switching protocol [15]-[19].

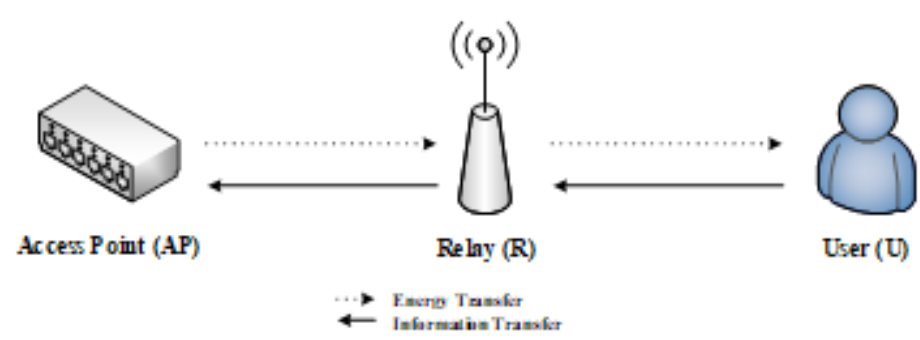

Figure 1. System model

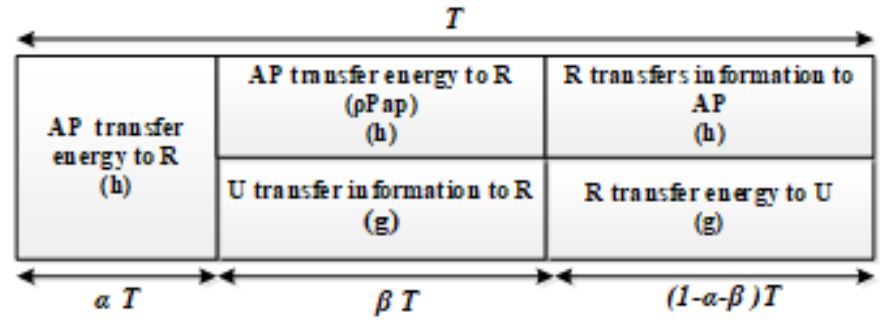

Figure 2. The power splitting protocol 


\section{THE SYSTEM PERFORMANCE}

In this part, the system performance in term of throughput and outage probability of the hybrid timepower switching protocols for the AF energy harvesting bidirectional relaying network is investigated for both delay-limited (DL) and delay-tolerant (DT) transmission modes. Let $x_{u}$ denote the transmitted signal from the user during the second phase, and $P_{u}$ denote the power of this signal. Then, the received signal at the relay $\mathrm{R}$ can be calculated by:

$y_{r}=\sqrt{P_{a p}} h x_{a p}+\sqrt{P_{u}} g x_{u}+n_{r}$

Where $\mathrm{x}_{\mathrm{ap}}$ and $\mathrm{x}_{\mathrm{u}}$ denote the transmitted signals from AP and the mobile user, respectively,

$\mathrm{P}_{\mathrm{u}}$ is the power at the user,

$\mathrm{P}_{\mathrm{r}}$ is the power at the relay,

$\mathrm{n}_{\mathrm{r}}, \mathrm{n}_{\mathrm{d}}$ are the additive white Gaussian noise (AWGN) at $R$ with zero mean and variance $\mathrm{N}_{0}$.

The harvesting energy $E_{r}$ at $R$ in the first and second interval time can be formulated as:

$E_{r}=\eta P_{a p}|h|^{2} \alpha T+\eta \rho P_{a p}|h|^{2} \beta T$

The transmission power of the $\mathrm{R}$ in the thirst interval is:

$P_{r}=\frac{E_{r}}{(1-\alpha-\beta) T}=\frac{\eta P_{a p}|h|^{2} \alpha T+\eta \rho P_{a p}|h|^{2} \beta T}{(1-\alpha-\beta) T}=k P_{a p}|h|^{2}$

where we denote $k=\frac{\eta(\alpha+\beta \rho)}{1-\alpha-\beta}$.

In the similar way, while the AP receives the information signal, the mobile user also receives the $\mathrm{RF}$ energy sent from the relay. By ignoring the noise energy, which is negligible as compared to the signal energy. The received signal at mobile user is $y_{u}=g x_{r}$. Hence, the energy harvested during this phase can be determined by:

$$
E_{u}=\eta|g|^{2} E_{r}=\eta|g|^{2}\left\{\eta P_{a p}|h|^{2} \alpha T+\eta \rho P_{a p}|h|^{2} \beta T\right\}
$$

So the transmit power of the user during the thirst phase is expressed as the below equation:

$P_{u}=\frac{E_{u}}{(1-\alpha-\beta) T}=\frac{\eta|g|^{2}\left\{\eta P_{a p}|h|^{2} \alpha T+\eta \rho P_{a p}|h|^{2} \beta T\right\}}{1-\alpha-\beta}=k \eta P_{a p}|h|^{2}|g|^{2}$

We select $(\alpha+\beta)<1$, because $\mathrm{P}_{\mathrm{R}}, \mathrm{P}_{\mathrm{u}}$ must be positive.

After precanceling the signal $\mathrm{x}_{\mathrm{ap}}$, the remaining part of the received signal in transmission phase at the relay is written as:

$y_{r}=\sqrt{1-\rho} \sqrt{P_{u}} g x_{u}+n_{r}$

In $\mathrm{AF}$ protocol, the signal transmitted by $\mathrm{R}$ during the second phase is:

$$
\xi=\frac{x_{r}}{y_{r}}=\sqrt{\frac{k P_{a p}|h|^{2}}{(1-\rho) P_{u}|g|^{2}+N_{0}}}
$$

Where $\xi$ : amplify factor.

The received signal at the user can be calculated as: 
$y_{u}=h \xi y_{r}+n_{u}=\underbrace{h \xi \sqrt{1-\rho} \sqrt{P_{u}} g x_{u}}_{\text {signal }}+\underbrace{h \xi n_{r}+n_{u}}_{\text {noise }}$

Delay limited transmission node.

The end to end signal to noise ratio (SNR) at the relay is:

$$
S N R_{A F}=\frac{\xi^{2}|h|^{2}(1-\rho) P_{u}|g|^{2}}{\xi^{2}|h|^{2} N_{0}+N_{0}}=\frac{|h|^{2}(1-\rho) P_{u}|g|^{2}}{|h|^{2} N_{0}+N_{0} / \xi^{2}}
$$

By substituting, we have:

$$
S N R_{A F}=\frac{k \eta(1-\rho) P_{a p}|h|^{4}|g|^{4}}{|h|^{2} N_{0}+N_{0} \frac{k \eta(1-\rho) P_{a p}|h|^{2}|g|^{4}+N_{0}}{k P_{a p}|h|^{2}}}=\frac{k^{2} \eta(1-\rho) \gamma_{0} X^{2} Y^{2}}{k X+k \eta(1-\rho) Y^{2}+\frac{1}{\gamma_{0} X}}
$$

Where we denote $X=|h|^{2}, Y=|g|^{2}, \gamma_{0}=\frac{P_{a p}}{N_{0}}$.

At the high SNR regime, we can use the following approximation:

$$
\frac{k X+k \eta(1-\rho) Y^{2}+\frac{1}{\gamma_{0} X}}{k X+k \eta(1-\rho) Y^{2}+\sqrt{\frac{k \gamma_{t h}}{\gamma_{0}}}} \approx 1
$$

Where $\gamma_{t h}=2^{2 R}-1, \mathrm{R}$ istarget rate.

The signal to noise ratio (SNR) at the relay $\mathrm{R}$ can be computed by the following equation:

$$
S N R_{A F} \approx \frac{k^{2} \eta(1-\rho) \gamma_{0} X^{2} Y^{2}}{k X+k \eta(1-\rho) Y^{2}+\sqrt{\frac{k \gamma_{t h}}{\gamma_{0}}}}
$$

The outage probability of the proposed system can be expressed as:

$$
\begin{aligned}
& P_{\text {out }}=\operatorname{Pr}\left(S N R_{A F}<\gamma_{\text {th }}\right)=\operatorname{Pr}\left(\frac{k^{2} \eta(1-\rho) \gamma_{0} X^{2} Y^{2}}{k X+k \eta(1-\rho) Y^{2}+\sqrt{\frac{k \gamma_{t h}}{\gamma_{0}}}}<\gamma_{\text {th }}\right) \\
& =\operatorname{Pr}\left(X^{2}<\frac{\gamma_{\text {th }}}{k \gamma_{0}}\right)+\operatorname{Pr}\left(Y^{2}<\frac{k \gamma_{\text {th }} X+\gamma_{\text {th }} \sqrt{\frac{k \gamma_{\text {th }}}{\gamma_{0}}}}{k^{2} \eta(1-\rho) \gamma_{0} X^{2}-k \eta(1-\rho) \gamma_{\text {th }}} \text { and } X^{2} \geq \frac{\gamma_{\text {th }}}{k \gamma_{0}}\right) \\
& P_{\text {out }}=\operatorname{Pr}\left(X<\sqrt{\frac{\gamma_{\text {th }}}{k \gamma_{0}}}\right)+\operatorname{Pr}\left(Y<\sqrt{\left.\frac{\gamma_{\text {th }}}{k \eta(1-\rho) \gamma_{0}\left[X-\sqrt{\frac{\gamma_{\text {th }}}{k \gamma_{0}}}\right.}\right]} \text { and } X \geq \sqrt{\frac{\gamma_{\text {th }}}{k \gamma_{0}}}\right) \\
& P_{1}=\operatorname{Pr}\left(X<\sqrt{\frac{\gamma_{\text {th }}}{k \gamma_{0}}}\right)=1-e^{-\lambda_{h} \sqrt{\frac{\gamma_{\text {th }}}{k \gamma_{0}}}}
\end{aligned}
$$


$P_{2}=\operatorname{Pr}\left(Y<\frac{\gamma_{t h}}{k \eta(1-\rho) \gamma_{0}\left[X-\sqrt{\frac{\gamma_{t h}}{k \gamma_{0}}}\right]}\right.$ and $\left.X \geq \sqrt{\frac{\gamma_{t h}}{k \gamma_{0}}}\right)=\int_{\sqrt{\frac{\gamma_{t h}}{k \gamma_{0}}}}^{\infty} f_{X}(x) d x \int_{0}^{z(x)} f_{Y}(y) d y$

$$
\begin{aligned}
& \text { Where } z(x)=\sqrt{\frac{\gamma_{\text {th }}}{k \eta(1-\rho) \gamma_{0}\left[x-\sqrt{\frac{\gamma_{\text {th }}}{k \gamma_{0}}}\right]}} \text {. } \\
& P_{2}=\int_{\sqrt{\frac{\gamma_{h h}}{k \gamma_{0}}}}^{\infty} f_{X}(x)\left[1-e^{-\lambda_{g} z(x)}\right] d x=e^{-\lambda_{h} \sqrt{\frac{\gamma_{h h}}{k \gamma_{0}}}}-\int_{\sqrt{\frac{\gamma_{h h}}{k \gamma_{0}}}}^{\infty} \lambda_{h} e^{-\lambda_{h} \sqrt{\frac{\gamma_{h h}}{k \gamma_{0}}}-\lambda_{g} z(x)} d x \\
& P_{\text {out }}=1-\int_{\sqrt{\frac{\gamma_{\text {th }}}{k \gamma_{0}}}}^{\infty} \lambda_{h} e^{-\lambda_{h} x-\lambda_{g}} \sqrt{\frac{\gamma_{\text {th }}}{k \eta(1-\rho) \gamma_{0}\left[x-\sqrt{\frac{\gamma_{h}}{k \gamma_{0}}}\right]}} d x
\end{aligned}
$$

Changing variable $t=x-\sqrt{\frac{\gamma_{t h}}{k \gamma_{0}}}$, we have:

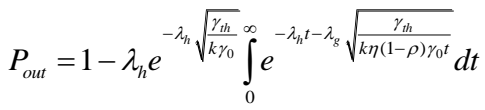

We use the Corollary (3.20) of Evans (24)

(24) Chaudhry MA, Zubair SM. Extended incomplete gamma functions with applications. J Math Anal Appl. 2002;274(2):725-745.

$$
P_{\text {out }}=1-\lambda_{h} e^{-\lambda_{h} \sqrt{\frac{\gamma_{\text {th }}}{k \gamma_{0}}}} \Gamma\left(1,0, \lambda_{g} \sqrt{\frac{\gamma_{\text {th }}}{k \eta(1-\rho) \gamma_{0}}}, \frac{1}{2}\right)=1-\frac{\lambda_{h} e^{-\lambda_{h} \sqrt{\frac{\gamma_{\text {th }}}{k \gamma_{0}}}}}{\sqrt{\pi}} G_{0,3}^{3,0}\left(\frac{\lambda_{g}^{2} \gamma_{\text {th }}}{4 k \eta(1-\rho) \gamma_{0}} \mid 0, \frac{1}{2}, 1\right)
$$

where $\Gamma(\bullet)$ : is the extended incomplete Gamma function $G_{p, q}^{m, n}(\square \mid \ldots)$ is the Meijer function (table of integral)

Finally, the throughput can be formulated as:

$$
\tau^{D L}=\left(1-P_{\text {out }}\right) R \frac{(1-\alpha-\beta) T}{T}=R(1-\alpha-\beta) \frac{\lambda_{h} e^{-\lambda_{h} \sqrt{\frac{\gamma_{h h}}{k \gamma_{0}}}}}{\sqrt{\pi}} G_{0,3}^{3,0}\left(\frac{\lambda_{g}^{2} \gamma_{\text {th }}}{4 k \eta(1-\rho) \gamma_{0}} \mid 0, \frac{1}{2}, 1\right)
$$

\section{Delay-Tolerant transmission} following:

In this section, we need to evaluate the ergodic capacity. We use the received signal SNR as the

$$
\begin{aligned}
& C_{A F}=\mathrm{E}_{|h|^{2},|g|^{2}}\left\{\log _{2}\left(1+S N R_{A F}\right)\right\} \\
& C_{A F}=\frac{1}{\ln 2} \int_{0}^{\infty} \frac{1-F_{S N R_{A F}}\left(\gamma_{t h}\right)}{1+\gamma_{t h}} d \gamma_{t h}
\end{aligned}
$$

Finally, ther ergodic capacitu can be formulated as: 
$C_{A F}=\frac{1}{\ln 2} \int_{0}^{\infty} \frac{1-F_{S N_{A A}}\left(\gamma_{t h}\right)}{1+\gamma_{t h}} d \gamma_{t h}=\frac{\lambda_{h}}{\sqrt{\pi} \ln 2} \int_{0}^{\infty} \frac{G_{0,3}^{3,0}\left(\frac{\lambda_{g}^{2} \gamma_{t h}}{4 k \eta(1-\rho) \gamma_{0}} \mid 0, \frac{1}{2}, 1\right) e^{-\lambda_{h} \sqrt{\frac{\gamma h h}{k \gamma_{0}}}}}{1+\gamma_{t h}} d \gamma_{t h}$

The throughput:

$\tau^{D T}=C_{A F} \frac{(1-\alpha-\beta) T}{T}=C(1-\alpha-\beta)$

\section{NUMERICAL RESULTS AND DISCUSSION}

In this paper, Monte Carlo simulation was conducted to verify the analysis developed in the previous section. For simplicity, in our simulation model, we assume that the source-relay and relaydestination distances are both normalized to unit value. For the delay-limited transmission mode, the outage probability, and achievable throughput are analyzed in details. On the other hand, the outage probability, and the ergodic capacity for the delay-tolerant transmission mode are proposed and demonstrated. Figure 3 and 4 plot the effect of $\rho$ on the outage probability and system throughput in the DL transmission mode, respectively. In this simulation, we set $P_{a p} / N_{0}=10 \mathrm{~dB}$ and $\alpha, \beta$ in the Figure 3 and 4 . Moreover, Figure 5 and 6 present the dependent of the outage probability and system throughput in the DL transmission mode on the $\eta$ of the proposed system. Furthermore, Figure 7 and 8 show the influence of the outage probability and system throughput in the DL transmission mode on the ratio $P_{a p} / N_{0}$. From the results, we can see that the analytical and simulation results well agree with each other.

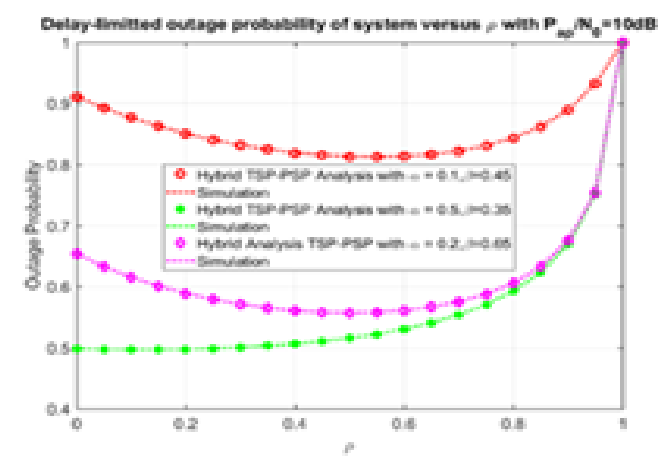

Figure 3. The outage probability versus $\rho$ for DL transmission mode

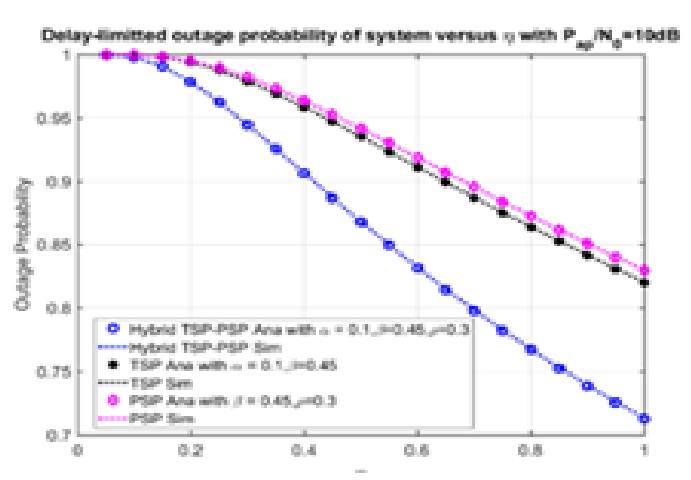

Figure 5. The outage probability versus $\eta$ for DL transmission mode

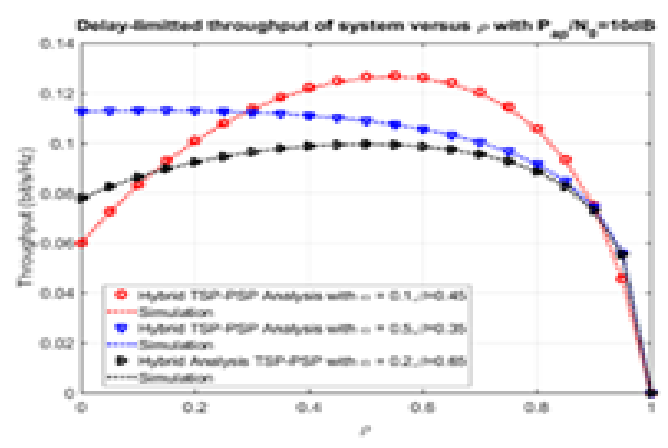

Figure 4. The throughput versus $\rho$ for DL transmission mode

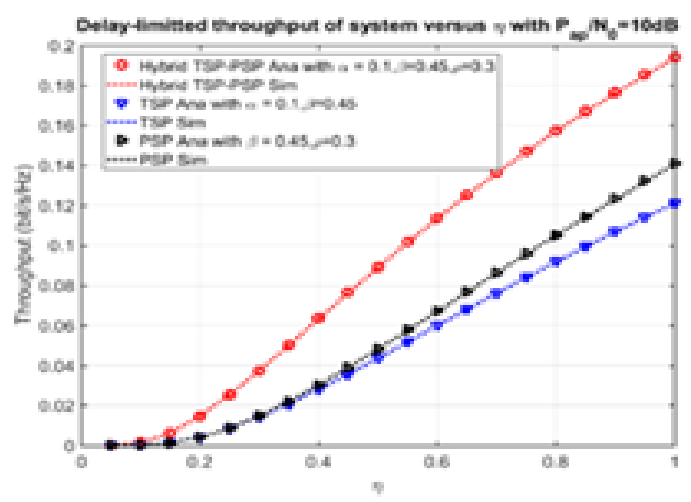

Figure 6. The throughput versus $\eta$ for DL transmission mode 
On another way, Figure 9 and 10 plot the effect of $\rho$ on the outage probability and system throughput in the DT transmission mode, respectively. In this simulation, we set Pap/N0 $=10 \mathrm{~dB}$ and $\alpha, \beta$ in the Figure 9 and 10. Moreover, Figure 11 and 12 present the dependent of the outage probability and system throughput in the DT transmission mode on the $\eta$ of the proposed system. Furthermore, Figure 13 and 14 show the influence of the outage probability and system throughput in the DT transmission mode on the ratio Pap/NO. From the results, we can see that the analytical and simulation results well agree with each other. In addition, the Figure 15 presents Comparison of the throughput versus Pap/N0 for DT and DL transmission modes for the both analytical and simulation cases.

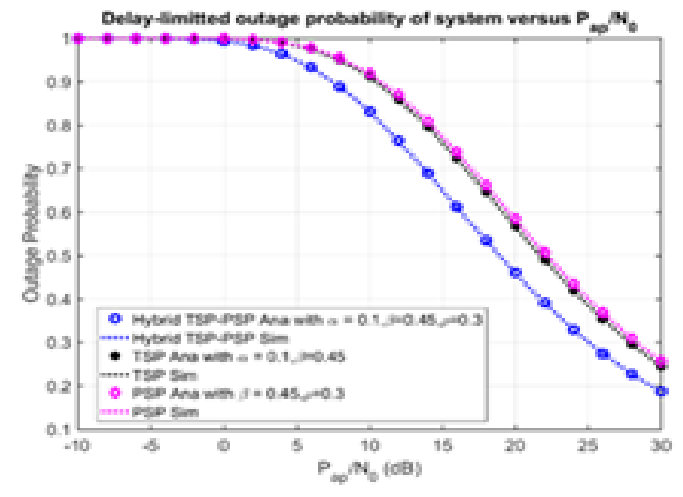

Figure 7. The outage probability versus $\mathrm{Pa} / \mathrm{N} 0$ for DL transmission mode

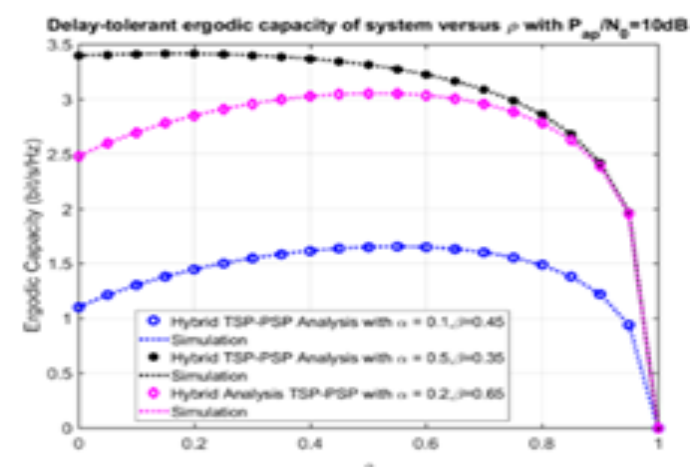

Figure 9. The outage probability versus $\rho$ for DT transmission mode

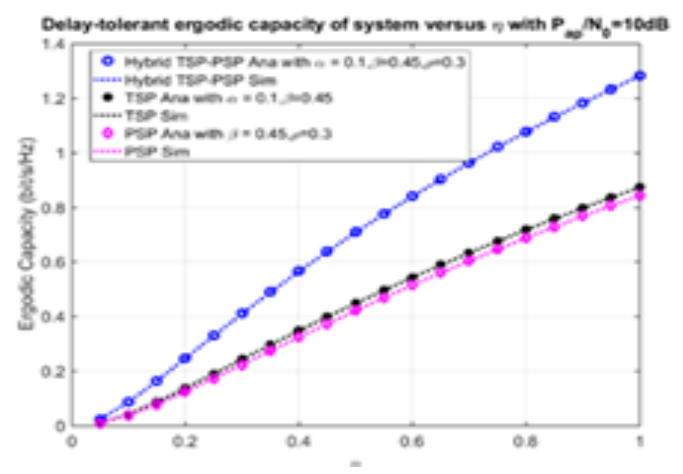

Figure 11. The ergodic capacity versus $\eta$ for DT

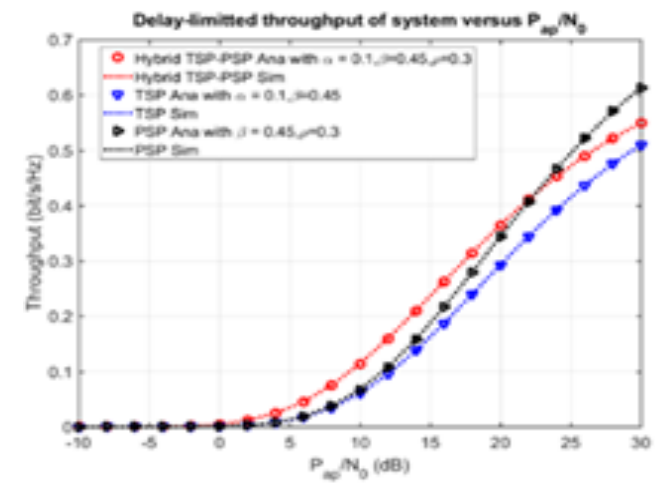

Figure 8. The throughput versus $\mathrm{Pa} / \mathrm{N} 0$ for DL transmission mode

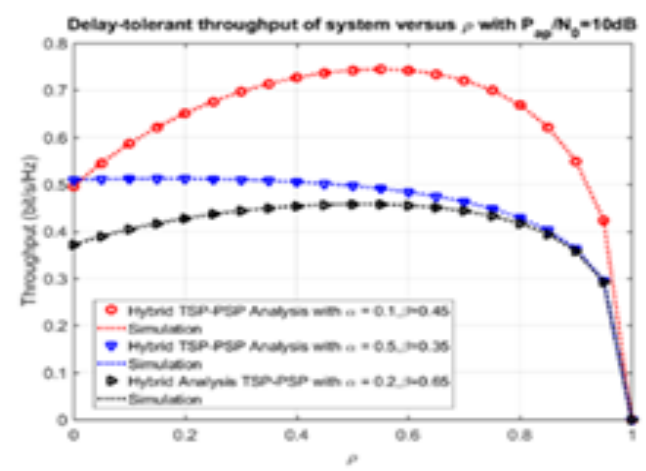

Figure 10. The throughput versus $\rho$ for DT transmission mode

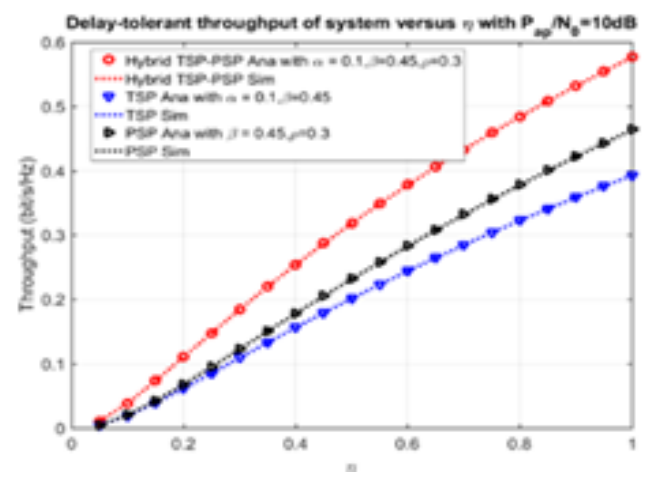

Figure 12. The throughput versus $\eta$ for DT 
transmission mode

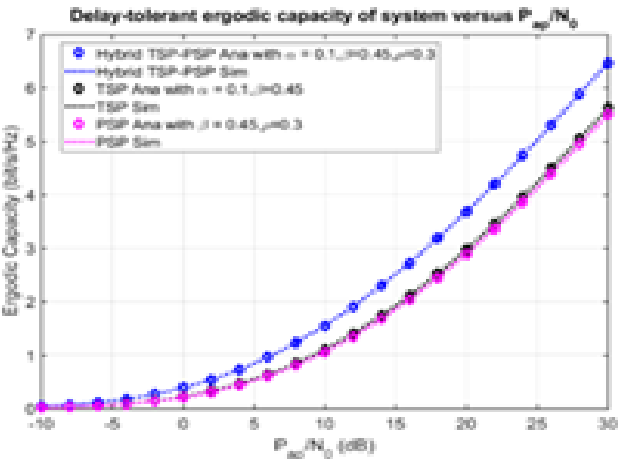

Figure 13. The ergodic capacity versus Pap/N0 for DT transmission mode

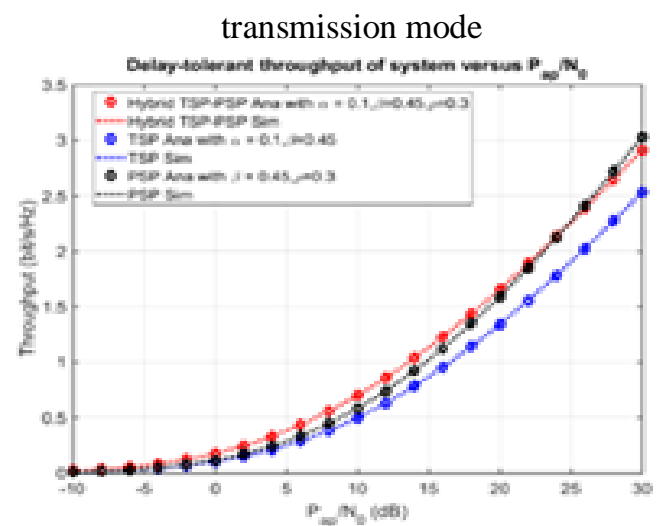

Figure 14. The throughput versus Pap/N0 for DT transmission mode

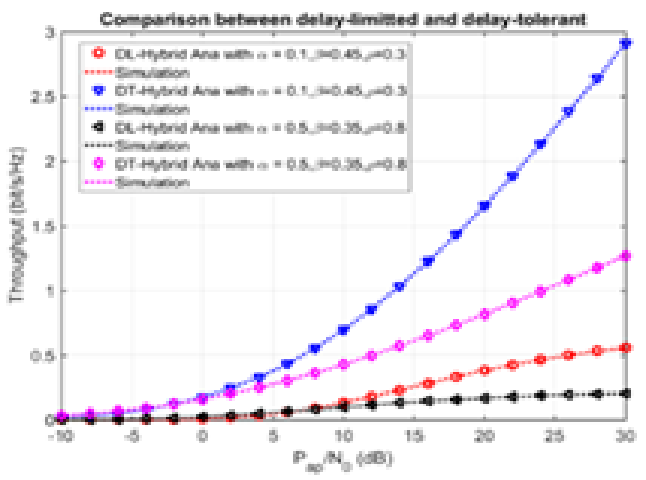

Figure 15. Comparison the throughput versus Pap/N0 for DT and DL transmission modes

\section{CONCLUSION}

In this paper, the hybrid time-power switching protocol of energy harvesting bidirectional relaying network is proposed and investigated. In order to analyze the system performance, analytical expressions for the outage probability, ergodic capacity and the throughput of the delay-limited and delay-tolerant transmission mode are investigated ad derived. The results show that the analytical mathematical and simulated results by Monte-Carlo simulation match for all possible parameter values for both schemes. The results could be provide the prospective solution for the communication network in the near future.

\section{REFERENCES}

[1] Bi, S., Ho, C. K., \& Zhang, R. (2015, 04). Wireless powered communication: Opportunities and challenges. IEEE Communications Magazine, 53(4), 117-125. doi:10.1109/mcom.2015.7081084

[2] Niyato, D., Kim, D. I., Maso, M., \& Han, Z. (2017). Wireless Powered Communication Networks: Research Directions and Technological Approaches. IEEE Wireless Communications, 2-11. doi:10.1109/mwc.2017.1600116

[3] Nasir, A. A., Zhou, X., Durrani, S., \& Kennedy, R. A. (2013, 07). Relaying Protocols for Wireless Energy Harvesting and Information Processing. IEEE Transactions on Wireless Communications, 12(7), 3622-3636. doi:10.1109/twc.2013.062413.122042

[4] Baidas, M. W., \& Alsusa, E. A. (2016, 02). Power allocation, relay selection and energy cooperation strategies in energy harvesting cooperative wireless networks. Wireless Communications and Mobile Computing, 16(14), 20652082. doi: $10.1002 / \mathrm{wcm} .2668$.

[5] Krikidis, I. (2014, 03). Simultaneous Information and Energy Transfer in Large-Scale Networks with/without Relaying. IEEE Transactions on Communications, 62(3), 900-912. doi:10.1109/tcomm.2014.020914.130825.

[6] Ding, Z., Krikidis, I., Sharif, B., \& Poor, H. V. (2014, 08). Wireless Information and Power Transfer in Cooperative Networks With Spatially Random Relays. IEEE Transactions on Wireless Communications, 13(8), 4440-4453. doi:10.1109/twc.2014.2314114.

[7] Mheich, Z., \& Savin, V. (2017, 03). Cooperative communication protocols with energy harvesting relays. 2017 Wireless Days. doi:10.1109/wd.2017.7918116. 
[8] Huang, C., Zhang, R., \& Cui, S. (2013, 08). Throughput Maximization for the Gaussian Relay Channel with Energy Harvesting Constraints. IEEE Journal on Selected Areas in Communications, 31(8), 1469-1479. doi:10.1109/jsac.2013.130811.

[9] Ahmed, I., Ikhlef, A., Schober, R., \& Mallik, R. K. (2013, 04). Joint Power Allocation and Relay Selection in Energy Harvesting AF Relay Systems. IEEE Wireless Communications Letters, 2(2), $239-242$. doi:10.1109/wcl.2013.012513.130007.

[10] Zeng, Y., Chen, H., \& Zhang, R. (2016, 05). Bidirectional Wireless Information and Power Transfer With a Helping Relay. IEEE Communications Letters, 20(5), 862-865. doi:10.1109/lcomm.2016.2549515.

[11] Gurakan, B., Ozel, O., Yang, J., \& Ulukus, S. (2013, 06). Energy cooperation in energy harvesting two-way communications. 2013 IEEE International Conference on Communications (ICC). doi:10.1109/icc.2013.6655023.

[12] Chaudhry, M., \& Zubair, S. (2002, 10). Extended incomplete gamma functions with applications. Journal of Mathematical Analysis and Applications, 274(2), 725-745. doi:10.1016/s0022-247x(02)00354-2 .

[13] J., J. E. (1979, 08). The H-Function with Applications in Statistics and Other Disciplines. Technometrics, 21(3), 392-393. doi:10.1080/00401706.1979.10489802.

[14] Table of Integrals, Series, and Products. (2015). doi:10.1016/c2010-0-64839-5.

[15] Tran Hoang Quang Minh. "Hybrid Time-Power Switching Protocol of Energy Harvesting Bidirectional Relaying Network: Throughput and Ergodic Capacity Analysis." TELKOMNIKA 16, no. 5 (10, 2018): 189. http://dx.doi.org/10.12928/telkomnika.v16i5.9118.

[16] Tin, Phu Tran, Tran Hoang Quang Minh, Tan N. Nguyen, and Miroslav Voznak. "System Performance Analysis of Half-Duplex Relay Network over Rician Fading Channel." TELKOMNIKA. 16, no. 1 (02, 2018): 189. doi:10.12928/telkomnika.v16i1.7491.

[17] Rashid, Tarique, Sunil Kumar, Akshay Verma, Prateek Raj Gautam, and Arvind Kumar. "Pm-EEMRP: Postural Movement Based Energy Efficient Multi-hop Routing Protocol for Intra Wireless Body Sensor Network (IntraWBSN)." TELKOMNIKA 16, no. 1 (02, 2018): 166. doi:10.12928/telkomnika.v16i1.7318.

[18] A. F. Morabito, "Power Synthesis of Mask-Constrained Shaped Beams Through Maximally-Sparse Planar Arrays," Telkomnika (Telecommunication Computing Electronics and Control), vol. 14, n. 4, pp. 1217-1219, 2016. 